\title{
FORÇA DAS RELAÇÕES: UM SURVEY EM CENTROS DE BELEZA
}

STRENGTH OF THE RELATIONSHIP: A SURVEY IN BEAUTY CENTERS

\author{
Recebido em 21.02.2018. Aprovado em 07.06.2018 \\ Avaliado pelo sistema double blind review \\ DOI: http://dx.doi.org/10.12712/rpca.v12i2.1234
}

\section{Rúbia Oliveira Corrêa}

Universidade Federal de Sergipe (UFS), Aracaju/SE, BRASIL

edrubia@gmail.com

\section{Luiz Marcos de Oliveira Silva}

Universidade Federal de Sergipe (UFS), Aracaju/SE, BRASIL

lmarcosufs@gmail.com

\section{Gustavo Dambiski Gomes de Carvalho}

Universidade Tecnológica Federal do Paraná (UTFPR), Curitiba/PR, BRASIL

gustavo.dambiski@gmail.com

\section{Patrícia Melo Sacramento}

Instituto Federal de Sergipe (IFS), Aracaju/SE, BRASIL

patriciamsacramento@gmail.com

\section{Resumo}

O presente estudo verificou a influência das características socioeconômicas e demográficas, assim como da duração, frequência e atributos do relacionamento na força da relação entre clientes e centros de beleza em Foz do Iguaçu PR. A pesquisa se caracteriza como descritiva e, em sua abordagem, classifica-se como quantitativa. A amostra foi do tipo não probabilística por acessibilidade, cujos dados foram coletados através de questionários e tratados por meio de frequências, teste de associação linear por linear, análise fatorial e regressão logística binária. Os resultados apontam relacionamento linear entre as variáveis independentes "duração da relação", "frequência de uso dos serviços", "sexo do cliente" e a variável dependente "força da relação empresa-cliente". Além disso, os atributos de relacionamento, em especial aqueles vinculados às competências individuais e profissionais do prestador do serviço, impactam positivamente para o fortalecimento da relação empresa-cliente na amostra pesquisada.

Palavras-chave: Força da Relação Empresa-Cliente. Duração da Relação. Frequência de Uso. Características Sociais e Demográficas. Atributos do Relacionamento.

\begin{abstract}
The present study verified the influence of socioeconomic and demographic characteristics, as well as the duration, frequency and attributes of the relationship in the strength of the relationship between clients and beauty centers in Foz do Iguaçu - PR. The research is presented as descriptive and, in its approach, is classified as quantitative. The sample was non-probabilistic for accessibility, whose data were collected through questionnaires and examined by means of frequencies, linear-by-linear association test, factor analysis and binary logistic regression. The results point to a linear relationship between the independent variables "client-client relationship duration", "meeting frequency", "client sex" and the dependent variable: the strength of the business-client relationship. In addition, relationship attributes, especially those related to service provider's individual and professional competencies, have a positive impact on strengthening the business-client relationship in the researched sample.
\end{abstract}

Keywords: Strength of the relationship firm-client. Length of relationship. Contact frequency. Social and Demographic Characteristics. Attributes of Relationship. 


\section{Introdução}

Núcleo do comportamento humano, as relações ocorrem em diversas esferas sociais. No âmbito comercial, a ideia de construir relacionamento com clientes, a fim de tirar vantagens dessa parceria, não era desconhecida dos primeiros empreendedores (WARD; DAGGER, 2007). Entretanto, por muitos anos, a maior parte das instituições comerciais centrou-se em adquirir novos clientes em detrimento de mantê-los. Tal postura era reflexo de estratégia de marketing de natureza transacional, em que as ações empresariais eram generalistas, focadas no curto prazo, e não havia interação entre organização e consumidores.

Nas últimas décadas, frente a ambientes de negócios mais competitivos, em que o número de ofertas para os consumidores é cada vez maior, modificou-se profundamente o comportamento dos clientes. Se, de um lado, o consumidor está mais informado, de outro é seduzido a cada instante por um outro produto ou marca (GRAZIANO et al., 2016). Neste novo cenário, a conquista $\mathrm{da}$ fidelidade dos clientes vem se estabelecendo como prioridade nas organizações (DEMO, 2010).

Diante do panorama apresentado, o investimento em estratégias de Marketing de Relacionamento (MR), com o objetivo de preservar e cultivar fortes relacionamentos com os clientes pode ser considerado fator primordial para as organizações (REIS et al., 2016). Essa nova filosofia de negócios prega a construção e a manutenção de relações duradouras entre clientes e fornecedores (D'ANGELO, SCHNEIDER; LARAN, 2006). Tais relações costumam promover ganhos consideráveis para ambas as partes (SHETH; PARVATIYAR, 2000), de modo que, fornecedores são beneficiados com lucros superiores (BESSEGATO; NEUHAUS, 2005), enquanto clientes podem desfrutar de segurança, sentimento de confiança, controle, redução dos riscos de compras e custos transacionais (GRONROOS, 2004).

Apesar da relevância da aplicação estratégica do MR para atração, manutenção e melhoramento das relações com clientes, a criação de uma estrutura conceitual para melhor compreendê-lo, assim como suas estruturas conceituais base, carecem de investigação acadêmica (WARD; DAGGER, 2007).

Ante o gap acima mencionado, o presente estudo verificou a influência das características socioeconômicas e demográficas, assim como da duração, frequência de uso e atributos do relacionamento na força da relação entre clientes e centros de beleza em Foz do Iguaçu - PR.
O mercado da beleza e estética está em franco crescimento, impulsionado pelos meios de comunicação, os quais trouxeram novos padrões de imagem e estilo que atingiram todas as camadas sociais e faixas etárias (GARBACCIO; OLIVEIRA, 2013). Pesquisa desenvolvida pela Associação Brasileira da Indústria de Higiene Pessoal, Perfumaria e Cosmético (ABIHPEC) constatou a expansão do referido setor e afirmou que o crescimento significativo dos salões de beleza, aqui denominados centros, também contribuiu para os bons resultados deste mercado em 2013 (BASÍLIO, 2014). Setor em ampliação e de expressivo fluxo de comunicação e relacionamentos informais, ele carece de estudos para melhor compreender aspectos da gestão e do comportamento do consumidor, justificando, assim, a escolha do ramo em que o estudo foi desenvolvido.

Considerando-se o que foi exposto, esta pesquisa adentra num campo duplamente instigante. De um lado, busca compreender estruturas conceituais base do MR e, de outro, analisar e propor um entendimento dos serviços de beleza através da elucidação de questões importantes sobre este mercado em visível expansão, uma vez que este setor é ainda pouco estudado pela comunidade científica brasileira.

O artigo foi divido em seções interdependentes, permitindo uma melhor compreensão das teorias que embasam a discussão. Para tanto, inicialmente é apresentada uma revisão teórica acerca dos temas centrais deste estudo. Em seguida, são registrados os procedimentos metodológicos. Por fim, apresenta-se a análise dos dados e as conclusões finais do trabalho.

\section{A Força da Relação Empresa-Cliente}

Diante da acirrada competitividade e da necessidade de melhorar o desempenho frente as oportunidades detectadas no mercado, as empresas estão se esforçando cada vez mais no sentido de estabelecer relacionamentos duradouros com seus parceiros de negócios e, em especial, com seus clientes (DEMO, 2014).

Estudo desenvolvido por Kumar et al. (2011) revelou que existe um efeito positivo da orientação para o mercado sobre o desempenho do negócio no que diz respeito tanto ao curto quanto ao longo prazo. Assim, Marketing de Relacionamento passou a ser visto como um novo paradigma, diferentemente da orientação meramente transacional. No MR, a conquista de clientes e sua retenção são questões organizacionais prioritárias (SHETH; PARVATIYAR, 2002 E PAYNE, 2006). Para Parvatiyar e Sheth (2000), essa nova abordagem de relacionamento 
comercial seria um processo contínuo em que atividades e programas cooperativos e colaborativos com clientes possibilitam a criação, ou ainda, a elevação do valor econômico mútuo a um custo reduzido.

É válido mencionar que os relacionamentos com parceiros de negócios podem ocorrer entre os mais diversos atores de uma cadeia de marketing (HOLMLUND, 2000). Essas relações costumam ser qualificadas por meio dos elementos: a) tipos de laços entre os atores; b) espécie de elos entre as atividades e c) tipo de ligações entre recursos. Esses elementos são essenciais para avaliar a natureza, o papel e a importância dessa espécie de relacionamento (FORD; GADDE; SNEHOTA, 2003).

Por meio dos relacionamentos com parceiros de negócios, uma organização pode tornar-se mais competitiva. Quando essa relação se estabelece com clientes, a empresa é capaz de melhor gerenciar questões que dizem respeito à fidelização do cliente externo, ao comprometimento do cliente interno com os objetivos de marketing da organização e ao compromisso do cliente intermediário com a qualidade, os prazos e as especificações definidas pelo mercado (GRAZIANO et al., 2016). A força dessa espécie de relacionamento, denominada neste estudo de "relacionamento empresa-cliente", foi definida por Kumar et al (2003) como a intensidade envolvida no relacionamento entre um fornecedor e seu cliente.

Estudos que investigam a força envolvida no relacionamento empresa-cliente trazem considerações bastante interessantes. A pesquisa de Ward e Dagger (2007), por exemplo, detectou que alguns clientes anseiam um relacionamento mais próximo com seus prestadores de serviços, e esse aspecto afeta significativamente a força do relacionamento percebida pelo cliente.

Estudo desenvolvido por Souza Neto e Mello (2009) visualiza um relacionamento como algo intrinsecamente dialógico, e, frente à diversidade do perfil dos clientes, cada um deles pode desejar uma forma diferente de relacionamento com uma mesma empresa. Assim, averiguaram que as relações de alta intensidade se caracterizam por interação com grande frequência, de longo prazo e de difícil rompimento do vínculo entre os envolvidos no processo, enquanto nos relacionamentos de baixa intensidade, os parceiros geralmente interagem com baixa frequência, focam prioritariamente o curto prazo nas interações e encontram poucas dificuldades para romper os vínculos que estabeleceram entre si.

O’Malley e Tynan (2000), por sua vez, salientaram que a maior parte das relações que envolvem empresas e clientes não costuma ser próxima e nem continuada. São, em geral, distantes e pontuais. Em muitos casos, o que se nomeia de relação não passa de uma associação assimétrica, desigual em termos de conhecimento, poder e recursos, isso porque talvez não seja exequível ou rentável manter relações próximas, pessoais e de longo prazo com todos os clientes (BARNES, 1997).

Ng, David e Dagger (2013) destacaram que relacionamentos fortes são fundamentais para as indústrias de serviços, uma vez que reduzem a rotatividade de clientes. Além disso, relacionamentos fortalecidos conduzem a atitudes leais e a carteiras de clientes. Tracey, Dagger e David (2011) contribuem com a investigação do tema quando revelam que os programas de fidelidade, investimentos em tecnologias e infraestrutura são algumas táticas empresariais que podem promover, de forma bem-sucedida, relacionamentos fortes entre clientes e fornecedores.

Os aspectos que promovem a força de uma relação comercial, apesar de serem pouco explorados academicamente, são de extrema relevância no "mundo dos negócios". Hausman (2001) revelou que entender o tema academicamente e gerencialmente resulta em insumos que subsidiarão informações e estratégias de planejamento, além de melhorias nos resultados relacionais entre clientes e fornecedores de serviços.

Miyamoto e Rexha (2004) destacaram que investir na relação com o cliente contribui para a promoção da imagem empresarial, visto que esses primeiros passam a perceber positivamente o empenho da firma no processo de solidificação e fortalecimento da relação com a clientela. Além disso, organizações que interagem e cooperam com seus clientes, desenvolvem relacionamentos baseados no comprometimento e na confiança, possibilitando dessa forma a geração de conhecimento de maneira conjunta (LARENTIS E'T AL 2014).

Morgan e Hunt (1994) asseguram que o estreitamento da relação empresa-cliente, principalmente aquelas solidificadas por meio da confiança e comprometimento entre as partes, resulta em uma menor probabilidade de dissolução. Nesse sentido, Scheer, Miao e Palmatier (2015) afirmam que quanto mais as partes estiverem interessadas em preservar o relacionamento e envolvidas entre si, menor o risco de rompimento da relação e maior a disposição delas a investir nesse relacionamento. 


\section{O Modelo Teórico e as Hipóteses de Pesquisa}

Ward e Dagger (2007), com base nas perspectivas teóricas do Marketing de Relacionamento, afirmam que existem circunstâncias favoráveis à formação do relacionamento empresa-cliente fortalecido. A citada relação pode ser desenvolvida por empreendimentos estabelecidos em qualquer ramo de atividade. No mais, a duração, a frequência com que faz uso dos serviços, características demográficas dos clientes e a importância que esses costumam atribuir a aspectos do relacionamento podem favorecer o desenvolvimento de relações comerciais em bases sólidas.

O modelo apresentado por Ward e Dagger (2007) foi base para este estudo, entretanto, com uma pequena adaptação, visto que aqui também foram consideradas a interferência das características socioeconômicas na intensidade da relação em questão, o que resultou no esboço a seguir:

Figura 1. O Modelo Teórico

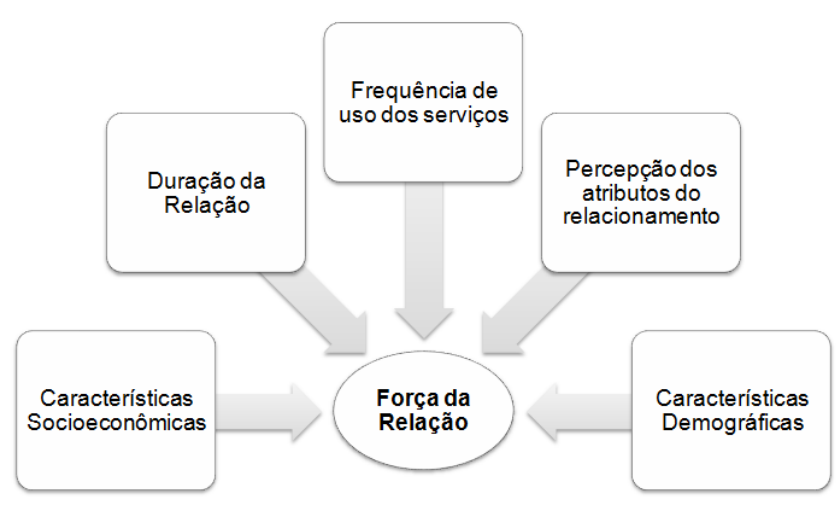

Fonte: Adaptado de Ward e Dagger (2007).

Sob uma ótica de estágios, as relações - aspecto relevante para modelo apresentado na figura 01 - são consolidadas por meio de uma série de episódios, de forma evolutiva, gradual e sequencial, sendo possível falar em ciclo de vida da relação. A questão temporal dos relacionamentos comerciais foi aqui abordada por meio da variável "duração da relação", definida como o período de tempo em que uma relação entre os agentes que promovem trocas, neste caso, cliente e prestador de serviço, perdura ou perdurou (COOIL et al., 2007).

Diante da temática, alguns estudos já detectaram uma relação direta entre a percepção do cliente acerca da força do relacionamento e duração da relação comercial (e.g. WARD; DAGGER, 2007; DAGGER; DANAHER E GIBBS, 2009). Isso implica dizer que quanto maior o tempo de relacionamento, maior seria a percepção da relação comercial por parte do cliente, o contrário também seria verdadeiro.

Para Nickels e Wood (1999), os relacionamentos duradouros entre empresas e clientes são planejados e fortalecidos por meio da aplicação dos mesmos princípios que regem as relações entre pessoas físicas. Dessa forma, esses relacionamentos duradouros são promovidos por meio de uma comunicação aberta, uma relação com base na confiança, honestidade, na ética, no contato frequente e na preocupação com o parceiro.

Compreender o quanto a duração de um relacionamento comercial poderá impactar na intensidade da relação desenvolvida entre cliente e fornecedor é de importância inquestionável. Frente à relevância acadêmica do tema e ao que foi exposto, é factível apresentar, neste momento, a primeira proposição testada pelo estudo em questão:

H1: Quanto maior o tempo que o cliente frequenta o centro de beleza, mais intensa será a percepção dele acerca da força da relação desenvolvida junto ao citado empreendimento.

Como segundo aspecto relevante da relação empresacliente, considera-se aqui a frequência com que $\mathrm{o}$ consumidor faz contato com seu prestador de serviço. A "frequência de uso dos serviços", variável investigada neste estudo, consiste no número de interações por período entre os parceiros de troca (PALMATIER et al. 2006).

Froemming (2001) afirma que as organizações de serviços e os encontros de serviços são inatos às relações da era pós-industrial. Reinartz e Kumar (2000) comentam que a frequência de contato entre cliente e a empresa é de grande importância para a construção da própria relação.

Cooil et al. (2007) asseveram que as relações evoluem com o passar do tempo e desenvolvem-se também diante de repetidas interações entre os parceiros de troca, ou seja, por meio da frequência do contato entre os atores. Nicholson, Compeau e Sethi (2001) salientam que os relacionamentos comerciais mais jovens requerem interações mais frequentes se comparados aos mais antigos, o que ocorre devido à necessidade de normatizar a nova relação.

Os encontros de serviços, em especial, são essenciais para a construção da imagem das empresas junto aos seus 
clientes; isso porque a impressão organizacional que a empresa imprime junto aos seus consumidores se dá no momento da prestação do serviço, da sua entrega, ou do contato pessoal (ZEITHAML; BITNER, 2003).

Por meio da mencionada interação, o consumidor constata a qualidade do serviço prestado, isso levaria à retenção de clientes já existentes e à atração de novos, à redução de custos, a uma imagem corporativa melhorada, à recomendação positiva boca a boca, e, finalmente, a uma maior lucratividade (LADHARI, 2009).

Estudo desenvolvido por Barnes (1997) expõe que o nível de contato entre consumidor e prestador de serviço é capaz de promover o fortalecimento da força da relação desenvolvida entre estes atores. Esta afirmativa sugere a existência de relação entre a frequência em que se faz uso de um determinado serviços e a força da relação que um tomador vem a desenvolver com seu prestador. Tal raciocínio resulta na segunda proposição do estudo:

H2: Quanto maior a frequência de uso dos serviços disponibilizados pelo centro de beleza, o cliente passa a perceber mais fortemente a força do relacionamento desenvolvida junto ao prestador de serviço.

Inúmeras são as pesquisas desenvolvidas acerca do Marketing de Relacionamento (e. g. SHETH; PARVATIYAR, 2002; ADJEI; CLARK, 2010), entretanto, poucas são aquelas em que se propôs examinar a relação entre as características socioeconômicas e demográficas e o Marketing de Relacionamento desenvolvido pelas organizações comerciais (WARD; DAGGER, 2007).

No presente estudo, as características socioeconômicas, outro aspecto relevante da relação empresa-cliente, é um construto teórico, que não pode ser diretamente medido, mas que se manifesta no nível de educação, ocupação e renda dos entrevistados (ALVES; SOARES, 2009). As características demográficas, por sua vez, podem se manifestar em indicadores relacionados ao sexo, idade, estado civil, região geográfica de residência ou de nascimento dos inqueridos, entre outros (CERQUEIRA; GIVISIEZ, 2004).

Diante da escassez de estudos que contemplam a relação mencionada, a presente pesquisa se propõe a examinar se a renda dos respondentes, o sexo, a idade e a distância entre a residência do cliente e o centro de beleza que frequenta impactam na força da relação entre clientes e fornecedores de serviços, desenvolvendo a seguinte hipótese:
H3: A renda do cliente $(\mathrm{H} 3 \mathrm{a})$, a idade $(\mathrm{H} 3 \mathrm{~b})$, o sexo $(\mathrm{H} 3 \mathrm{c})$, bem como a distância entre a residência deste $\mathrm{e}$ o centro de beleza que frequenta (H3d), afetam a força do relacionamento desenvolvida entre consumidores e centros de beleza.

Assim como as características socioeconômicas, demográficas e a duração dos relacionamentos, os atributos de relacionamento são aqui considerados aspectos relevantes para o fortalecimento da relação empresacliente.

Atributos de relacionamento foram operacionalmente definidos como aquilo que é próprio, inerente ao ato de relacionar-se (WARD; DAGGER, 2007). No presente estudo, são averiguados atributos básicos de uma relação: empatia, reciprocidade, confiança, amizade, reconhecimento, atenção, compreensão, tempo para ouvir, compromisso com o cliente e valores comuns.

Barry e Johnson (2008) afirmam que a força do relacionamento é influenciada por fatores sociais. Ward e Dagger (2007), por sua vez, informam que o desenvolvimento, a manutenção e o fortalecimento de um relacionamento vão depender da percepção do cliente acerca da importância das dimensões relacionais básicas, a exemplo das mencionadas anteriormente.

Vale ressaltar que, na prestação de serviço, ramo de atividade central neste estudo, a figura do prestador é consagrada como o meio mais seguro, capaz de promover relações mais fortes e duradouras com consumidores. No mais, os benefícios relacionais são percebidos pelos consumidores independentemente da intensidade de contato entre eles (MOTA; FREITAS, 2008).

Gwinner et al. (1998), pioneiros nas pesquisas que tratam de benefícios relacionais, já afirmavam que os consumidores, diante de suas relações com provedores de serviços, buscam satisfação e benefícios relacionais como laços sociais, confiança e tratamentos especiais. Sendo que os benefícios relacionais superam em importâmcia a busca da satisfação quando analisado o relacionamento empresa-cliente.

Mediante as afirmações anteriores, e a importância dada aos benefícios relacionais nas transações comerciais, surge a necessidade de testar a hipótese descrita a seguir:

H4: A percepção do cliente acerca da importância dos atributos de relacionamento desenvolvidos pelo seu fornecedor influenciará na força da relação desenvolvida entre eles. 
Mediante as proposições apresentadas, possíveis respostas ao problema dessa pesquisa, finaliza-se esta seção e, na sequência, são apresentados os procedimentos metodológicos deste estudo.

\section{Metodologia}

A presente pesquisa é caracterizada como Levantamento (Survey), visto que fornece uma descrição quantitativa de atitudes e opiniões de uma população por meio de uma amostra desta (CRESWELL, 2003). Sua unidade de análise é organizacional.

O estudo em questão foi classificado como descritivo, pois se preocupou em compreender a influência das características socioeconômicas e demográficas, assim como da duração, frequência e atributos do relacionamento, na força da relação entre clientes e centros de beleza em Foz do Iguaçu - PR.

Em sua abordagem, a presente pesquisa classifica-se como quantitativa, uma vez que se utilizou da quantificação para coletar e tratar as informações que subsidiaram o estudo em questão. Quanto à dimensão tempo, a pesquisa tem corte transversal, assim, o evento foi observado mediante um "corte" temporal.

A amostra foi da espécie não probabilística por acessibilidade, composta por 225 clientes maiores de 18 anos que frequentam os centros de beleza situados em Foz do Iguaçu. Para os fins deste estudo, os dados foram coletados por meio de questionários estruturados, composto por 27 questões, as quais foram redigidas com base nas variáveis da pesquisa, ou seja, características socioeconômicas e demográficas dos frequentadores dos centros de beleza, duração da relação, frequência de uso dos serviços, força da relação empresa-cliente e percepção dos clientes acerca da importância dos atributos do relacionamento.

A variável "características socioeconômicas" foi operacionalizada por meio do indicador renda dos respondentes. As "características demográficas" foram operacionalizadas por meio dos seguintes indicadores: sexo, idade e distância entre a residência do cliente e o centro de beleza que frequenta. A "duração da relação" e a "frequência de uso dos serviços" foram mensuradas por meio de indicadores de tempo.

A "força da relação entre empresa-cliente" foi operacionalizada por meio de uma escala semântica bipolar de 0 a 7 . O ponto 0 (zero) representa o indicador "ausência de relacionamento entre o respondente e o centro de beleza frequentado". Já os indicadores que representam os demais pontos $(1,2,3,4,5,6$ e 7$)$ foram utilizados pelos clientes que perceberam a existência de relação com seus fornecedores, sendo este intervalo, respectivamente, representado pelos indicadores: relação muito fraca, fraca, pouco fraca, nem fraca nem forte, pouco forte, forte e muito forte.

A percepção acerca da importância dos "atributos do relacionamento" foi medida através de uma escala de importância que varia de 1 a 5 . O ponto 1 (um) simboliza que os indicadores (empatia, reciprocidade, confiabilidade, relação amigável, competência para reconhecer a importância do cliente, capacidade de ser atencioso, habilidade para compreender as necessidades do cliente, tempo para ouvir o cliente, compromisso com as atividades desenvolvidas e respeito aos valores dos clientes) "não são importantes" para relação entre cliente e seu centro de beleza. Os demais pontos (2, 3, 4 e 5) representam que os indicadores, assim classificados, são considerados, respectivamente, como: pouco importantes, moderadamente importantes, importantes e muito importantes para a relação desenvolvida entre clientes e centros de beleza.

O tratamento dos dados foi realizado por meio de frequências (absolutas, relativas e acumuladas) para evidenciar dados gerais acerca da variável dependente (força da relação). O teste qui-quadrado de associação linear por linear averiguou a relação da variável dependente com as independentes: características socioeconômicas, demográficas, frequência de uso e duração da relação. Os atributos de relacionamento foram reduzidos por meio da análise dos componentes principais (análise fatorial exploratória), que se utilizou da rotação ortogonal do tipo Varimax com normalização Kaiser. A relação entre esta última variável e a força da relação foi constatada por meio de análise de logística binária.

\section{Apresentação e Análise dos Dados}

\section{Força da Relação entre Clientes e Centros de Beleza em Foz do Iguaçu - PR}

Frente aos dados coletados, foi possível averiguar, conforme descrito na tabela 1, que alguns poucos entrevistados (4,9\%) declararam não possuir relação com os centros de beleza que frequentam. Um percentual maior $(18,6 \%)$ declara relacionar-se de forma fraca com seus prestadores de serviço. Uma parte maior dos entrevistados $(25,8 \%)$ percebe que desenvolveu um relacionamento de força neutra (nem forte nem fraca) junto aos centros 
de beleza que frequentam. Entretanto, a maioria dos inquiridos $(50,7 \%)$ percebe como forte a força da relação desenvolvida com as empresas objeto do estudo.

Tabela 1. Força da Relação Empresa-Cliente

\begin{tabular}{|c|l|l|l|l|}
\hline \multicolumn{2}{|c|}{} & $\begin{array}{l}\text { Frequência } \\
\text { Absoluta }\end{array}$ & $\begin{array}{l}\text { Frequência } \\
\text { Relativa }\end{array}$ & $\begin{array}{l}\text { Frequência } \\
\text { Acumulada }\end{array}$ \\
\hline \multirow{6}{*}{} & $\begin{array}{l}\text { Não há } \\
\text { Relação }\end{array}$ & 11 & 4,9 & 4,9 \\
\cline { 2 - 5 } & $\begin{array}{l}\text { Muito } \\
\text { Fraca }\end{array}$ & 12 & 5,3 & 10,2 \\
\cline { 2 - 5 } & Fraca & 18 & 8,0 & 18,2 \\
\cline { 2 - 5 } & $\begin{array}{l}\text { Pouco } \\
\text { Fraca }\end{array}$ & 12 & 5,3 & 23,6 \\
\cline { 2 - 5 } & $\begin{array}{l}\text { Nem } \\
\text { Forte } \\
\text { nem } \\
\text { Fraca }\end{array}$ & 58 & 25,8 & 49,3 \\
\cline { 2 - 5 } & $\begin{array}{l}\text { Pouco } \\
\text { Forte }\end{array}$ & 37 & 16,4 & 65,8 \\
\cline { 2 - 5 } & Forte & 53 & 23,6 & 89,3 \\
\cline { 2 - 5 } & $\begin{array}{l}\text { Muito } \\
\text { Forte }\end{array}$ & 24 & 10,7 & 100,0 \\
\cline { 2 - 5 } & Total & 225 & 100,0 & \\
\hline
\end{tabular}

Fonte: Pesquisa de Campo, 2014.

\section{A Duração do Relacionamento e sua Associação com a Força da Relação Empresa-Cliente}

A associação realizada entre a variável categórica dependente (força da relação) e a independente (duração da relação) está descrita na tabela 2 .

Tabela 2 . Teste de qui-quadrado (duração da relação versus força da relação empresa-cliente)

\begin{tabular}{|l|l|l|l|c|}
\hline & Valor & df & Sig. Assint. (2 lados) & H1 \\
\hline $\begin{array}{l}\text { Associação } \\
\text { Linear por } \\
\text { Linear }\end{array}$ & 19,917 & 1 & 0,000 & Aceita \\
\hline $\begin{array}{l}\text { N de Casos } \\
\text { Válidos }\end{array}$ & 225 & & & \\
\hline
\end{tabular}

Fonte: Pesquisa de Campo, 2014.

A tabela 2 mostra o qui-quadrado de Associação Linear por Linear inferiores a 0,05 , sendo possível afirmar que as variáveis acima analisadas estão relacionadas/ associadas e são dependentes uma da outra. Percebeu- se que há evidências de associação significante entre: a) Relacionamentos de menor duração (até 3 anos) e relação empresa-cliente fraca ou pouco fraca; b) Relacionamentos empresa-cliente mais maduros (acima de 7 anos) e relação empresa-cliente muito forte.

A relação de dependência entre as variáveis aqui destacadas já foi detectada em outros estudos (eg. WARD; DAGGER, 2007; DAGGER; DANAHER E GIBBS, 2009). Os fatos aqui apresentados evidenciam uma relação de dependência direta entre as variáreis duração de relacionamento e percepção da força da relação comercial por parte do cliente. Assim, a hipótese H1 do estudo foi confirmada, isto é, a duração da relação está significativamente associada à força da relação empresa-cliente.

\section{Frequência de Uso dos Serviços e sua Associação com a Força da Relação Empresa-Cliente}

O teste qui-quadrado de Associação Linear também foi usado para averiguar a associação entre as variáveis frequência de uso dos serviços e percepção da força da relação empresacliente. Na tabela 3 é possível averiguar com detalhes essa associação.

Tabela 3 . Teste de qui-quadrado (frequência de uso versus força da relação empresa-cliente)

\begin{tabular}{|l|c|c|c|c|}
\hline & Valor & df & $\begin{array}{c}\text { Sig. } \\
\text { Assint. } \\
\text { (2 lados) }\end{array}$ & H2 \\
\hline $\begin{array}{l}\text { Associação } \\
\text { Linear por } \\
\text { Linear }\end{array}$ & 17,61 & 1 & 0,000 & Aceita \\
\hline $\begin{array}{l}\text { N de Casos } \\
\text { Válidos }\end{array}$ & 225 & & & \\
\hline
\end{tabular}

Fonte: Pesquisa de campo, 2014.

Segundo Souza Neto e Mello (2009), relações fortes, de alta intensidade, em geral, caracterizam-se por interação com grande frequência, de longo prazo e de difícil rompimento do vínculo entre os envolvidos no processo. Já os relacionamentos de baixa intensidade, relações fracas, caracterizam-se, na maior parte das vezes, por interações de baixa frequência, com foco no curto prazo e vínculos 
de fácil rompimento. A pesquisa desenvolvida por Ward e Dagger (2007) constatou uma relação de dependência direta entre as variáveis força da relação e frequência no uso dos serviços diante de interações entre: pacientes e médicos, clientes e cabeleireiros e clientes e cinemas. Já a relação correntista e bancos não evidenciou associação entre frequência de uso dos serviços e fortalecimento da relação empresa-cliente.

$\mathrm{Na}$ pesquisa aqui apresentada, frente a amostra examinada e mediante a significância estatística da Associação Linear por Linear (valor-p $<0,0001$ ), foi possível comprovar a hipótese $\mathrm{H} 2$ proposta pelo estudo. Após a análise dos resultados é possível asseverar que há associação significativa entre: a) frequência diária e a percepção de uma relação muito forte entre centros de beleza e seus clientes; b) frequência semanal e a percepção de uma relação empresa-cliente forte ou muito forte e c) frequência semestral e percepção de uma relação muito fraca entre estabelecimentos comerciais e seus clientes.

É válido ressaltar que, diante da diversidade de perfis de clientes, cada um se relaciona com seus prestadores de serviço conforme a própria conveniência (SOUZA NETO; MELLO, 2009). Cientes de que a maior parte das relações que envolvem empresas e clientes não costuma ser próxima e nem continuada, mas sim distantes e pontuais (O'MALLEY; TYNAN, 2000), acredita-se que, além da predisposição dos atores envolvidos, o ramo de atividade e a natureza do relacionamento impactam na relação de dependência entre as variáveis frequência de uso dos serviços e força da relação percebida.

\section{Aspectos Socioeconômicos e Demográficos e suas Associações com a Força da Relação Empresa-Cliente}

A variável características socioeconômicas é, neste estudo, representada pelo indicador classe social dos respondentes. O teste qui-quadrado de associação linear por linear foi utilizado para verificar a relação de dependência entre classe social (variável independente) e força da relação empresacliente (variável dependente). $\mathrm{O}$ teste em questão não apresentou significância estatística que pudesse comprovar a relação de dependência entre renda e força da relação empresa-cliente. Tal afirmativa pode ser comprovada diante da tabela 4 , que revela o valor de 0,741 para a significância estatística, valor muito superior ao valor crítico de 0,05 , rejeitando a hipótese H3a do estudo.
Tabela 4 . Teste de qui-quadrado (classe social versus força da relação empresa-cliente)

\begin{tabular}{|l|l|l|l|l|}
\hline & Valor & df & $\begin{array}{l}\text { Sig. } \\
\text { Assint. (2 } \\
\text { lados) }\end{array}$ & H3a \\
\hline $\begin{array}{l}\text { Associação } \\
\text { Linear por } \\
\text { Linear }\end{array}$ & 0,11 & 1 & 0,741 & Rejeitada \\
\hline $\begin{array}{l}\text { N de Casos } \\
\text { Válidos }\end{array}$ & 225 & & & \\
\hline
\end{tabular}

Fonte: Pesquisa de campo, 2014.

A relação de dependência entre características demográficas (idade e distância entre a residência do cliente e o centro de beleza que frequenta) e percepção da força da relação empresa-cliente também foi analisada por meio da aplicação do teste qui-quadrado de associação linear por linear. Diante dos seus valores críticos, superiores a 0,05 , foi possível afirmar que não existe relação de dependência ou associativa significantes entre força da relação empresa - cliente e idade (H3b) ou distância entre a residência do cliente e os centros de beleza frequentados (H3d).

A relação da variável dependente com o "sexo do respondente" é um caso a ser averiguado de forma particular. $\mathrm{Na}$ tabela 5, o teste de Associação Linear por Linear (valor-p $=0,009<0,005)$ imprime que há associação significativa entre algumas células resultantes do cruzamento entre sexo do respondente e a força da relação desenvolvida entre ele e o centro de beleza. Assim, a hipótese $\mathrm{H} 3 \mathrm{c}$ do estudo foi confirmada, enquanto as hipóteses H3a, H3b e H3d foram rejeitadas.

Tabela 5 . Testes de qui-quadrado (Sexo versus Força da Relação)

\begin{tabular}{|l|l|l|l|l|}
\hline & Valor & df & $\begin{array}{l}\text { Sig. Assint. } \\
(2 \text { lados })\end{array}$ & H3c \\
\hline $\begin{array}{l}\text { Associação } \\
\text { Linear por } \\
\text { Linear }\end{array}$ & 6,764 & 1 & 0,009 & Aceita \\
\hline $\begin{array}{l}\text { N de Casos } \\
\text { Válidos }\end{array}$ & 225 & & & \\
\hline
\end{tabular}

Fonte: Pesquisa de campo, 2014.

A análise dos resultados da interação supracitada confirma que há associação significativa entre: a) Mulheres e relação pouco e muito forte com seu centro de beleza; b) Homens e ausência de força na relação com seu prestador de serviço.

De acordo com Adjei e Clark (2010), nem todos os clientes possuem predisposição para relacionarem-se com seus 
prestadores de serviços, entretanto, aqueles mais dispostos, no caso em destaque as respondentes do sexo feminino, tenderão a desenvolver relações mais profundas e fiéis diante do prestador.

\section{A Percepção dos Atributos de Relacionamento e 0 Impacto na Força da Relação Empresa-Cliente}

Os indicadores da variável atributos do relacionamento (empatia, reciprocidade, confiabilidade, relação amigável, competência para reconhecer a importância do cliente, capacidade de ser atencioso, habilidade para compreender as necessidades do cliente, tempo para ouvir o cliente, compromisso com as atividades desenvolvidas e respeito aos valores dos clientes) foram reduzidos por meio de fatorial a dois componentes.

Diante do valor da medida Kaiser-Meyer-Olkin de adequação de amostragem $(0,820)$, percebe-se que o teste supracitado fora adequado para tratar os dados relacionados à variável Atributos do Relacionamento. A significância do teste de esfericidade de Bartlett $(0,000)$ é bastante pequena, isso confirma que o método fatorial é apropriado para tratar os dados em questão.

Os componentes extraídos diante do método de extração da Análise do Componente Principal (empatia, reciprocidade, confiabilidade, competência para reconhecer a importância do cliente, capacidade de ser atencioso, compromisso com as atividades desenvolvidas e respeito aos valores dos clientes) apresentaram em comunalidades de variáveis significativas, ou seja, com percentuais de variâncias superiores a 0,5.

Considerando os eigenvalues - valores próprios iniciais - dois fatores com valores próprios superiores a 1 (em destaque) foram extraídos. Esses fatores explicam 66,21\% da variabilidade total do modelo.

$\mathrm{Na}$ sequência, a tabela 6 traz a carga fatorial dos componentes extraídos após rotação da matriz principal por meio do método Varimax com normalização de Kaiser. Os novos fatores, 01 e 02, foram nomeados, respectivamente, de Aspectos de Competências Individuais e Competências Profissionais.

Os Aspectos de Competências Individuais foram compostos pelos indicadores: Empatia, Reciprocidade e Confiabilidade. As Competências Profissionais foram reduzidas aos indicadores: Competência para reconhecer a importância do cliente, Capacidade de ser atencioso, Compromisso com as atividades desenvolvidas e Respeito aos valores dos clientes.
Tabela 6 . Matriz de componente rotativa ${ }^{a}$

\begin{tabular}{|l|l|l|}
\hline \multirow{2}{*}{} & \multicolumn{2}{|l|}{ Componentes } \\
\cline { 2 - 3 } & $\begin{array}{l}\text { Competências } \\
\text { Profissionais }\end{array}$ & $\begin{array}{l}\text { Competências } \\
\text { Individuais }\end{array}$ \\
\hline Empatia &, 075 &, $\mathbf{8 7 2}$ \\
\hline Reciprocidade &, 267 &, 847 \\
\hline Confiabilidade &, 374 &, 708 \\
\hline $\begin{array}{l}\text { Competência } \\
\text { para reconhecer } \\
\text { a importância do } \\
\text { cliente }\end{array}$ &, 721 &, 285 \\
\hline $\begin{array}{l}\text { Capacidade de ser } \\
\text { atencioso }\end{array}$ &, 701 &, 280 \\
\hline $\begin{array}{l}\text { Compromisso } \\
\text { com as atividades } \\
\text { desenvolvidas }\end{array}$ &, 733 &, 247 \\
\hline $\begin{array}{l}\text { Respeito aos } \\
\text { valores dos } \\
\text { clientes }\end{array}$ &, 818 & \\
\hline $\begin{array}{l}\text { Método de extração: Análise do Componente } \\
\text { principal. } \\
\text { Método de rotação: Varimax com normalização de } \\
\text { Kaiser. } \\
\text { a. Rotação convergida em 3 interações. }\end{array}$ \\
\hline
\end{tabular}

Fonte: Pesquisa de Campo, 2014.

Após a redução dos fatores, a relação entre estes e a variável dependente (força da relação empresa-cliente) foi visualizada mediante a técnica Regressão de Logística. O método em destaque determina a probabilidade de ocorrência das relações empresa-cliente fracas e fortes. $\mathrm{Na}$ tabela 7, têm-se os outputs da Regressão das Variáveis que compuseram o modelo. A estatística de $W$ ald informa que os coeficientes B encontrados diferem significativamente de zero, assim, de fato, os previsores estão contribuindo de modo expressivo para a explicação do modelo de previsão proposto. Assim, a hipótese 4 (H4) deste estudo foi confirmada.

A Exp (b) indica que a percepção da importância do fator Competências Individuais aumenta em 1,5 vezes a probabilidade de o cliente desenvolver uma relação de intensidade forte com seu centro de beleza, desde que essas competências sejam postas em prática pelo prestador. Já a percepção da importância do fator Competências Profissionais apresenta-se mais impactante, aumenta em 2,03 vezes as chances de o cliente desenvolver uma relação forte com seu fornecedor. 
Tabela 7 . Outputs da Regressão das Variáveis do Modelo (Força da Relação versus Atributos do Relacionamento)

\begin{tabular}{|c|c|c|c|c|c|c|c|}
\hline & & $\mathrm{B}$ & S.E. & Wald & $\mathrm{df}$ & Sig. & $\operatorname{Exp}(B)$ \\
\hline \multirow{3}{*}{ Etapa $1^{a}$} & Competências Individuais & , 436 & ,166 & 6,890 & 1 & ,009 & \multirow{3}{*}{$\begin{array}{l}2,038 \\
3,775\end{array}$} \\
\hline & Competências Profissionais & ,712 & ,172 & 17,170 & 1 &, 000 & \\
\hline & Constante & 1,328 & 177 & 56,470 & 1 &, 000 & \\
\hline
\end{tabular}

Fonte: Pesquisa de Campo, 2014.

Já é sabido que consumidores buscam satisfação e benefícios relacionais junto a seus prestadores de serviços. Sendo que os benefícios relacionais superam em importância a busca da satisfação quando analisado o relacionamento empresacliente (GWINNER et al., 1998).

Os dados da tabela 7 mostram que os atributos de relacionamento, em especial aqueles vinculados às competências individuais e profissionais do prestador do serviço, impactam positivamente para o fortalecimento da relação empresacliente da amostra pesquisada. Os resultados encontrados estão em consonância com a literatura, a qual afirma que a força do relacionamento é influenciada por fatores sociais - como medido pela qualidade do relacionamento -, conforme foi exposto na pesquisa desenvolvida por Barry e Johnson (2008). Para Ward e Dagger (2007) os atributos relacionais impactam não apenas na força da relação densenvolvida entre empresa e clientes, mas também no processo de desenvolvimento e manutenção dessa relação.

\section{Conclusões}

O presente estudo verificou a influência das características socioeconômicas e demográficas, assim como da duração, frequência e atributos do relacionamento, na força da relação entre clientes e centros de beleza em Foz do Iguaçu - PR.

Frente à análise dos dados, constatou-se que a maioria dos inquiridos percebe como forte a relação desenvolvida com os centros de beleza que frequentam. Estudo desenvolvido por Barnes (2002) afirma que os relacionamentos costumam ser mais próximos e informais entre clientes e pequenas organizações, que seria o caso da maior parte dos centros de beleza situados em Foz do Iguaçu. Já a interação entre clientes e organizações de grande porte é mais rara, uma vez que seus clientes costumam se ater apenas aos aspectos transacionais da interação.

No mais, alguns tipos de serviços podem ser mais propícios para promover fortes relações empresa-cliente (LOVELOCK; PATTERSON; WALKER, 2007). Seria de esperar, por exemplo, que as relações mais estreitas se formam com o médico de família do que com empreendimentos de fast-food (DAGGER; DANAHER; GIBBS, 2009).

Detectou-se também uma associação direta entre as variáreis duração do relacionamento e percepção da força na relação comercial por parte do cliente. Diante dessa constatação, conclui-se que, para a amostra em questão, quanto maior o tempo que o cliente frequenta o centro de beleza, mais intensa será a percepção dele acerca da força da relação desenvolvida junto ao citado empreendimento. Isso veio a confirmar a primeira hipótese (H1) investigada neste estudo.

É possível ainda concluir que, junto ao público pesquisado, existe uma relação associativa entre as variáveis: frequência de uso dos serviços e força da relação empresa-cliente, em particular, uma associação significativa entre as maiores frequências de uso do serviço (diário, semanal) e a percepção mais fortalecida da relação empresa-cliente. Além disso, detectou-se associação entre a frequência semestral e relação muito fraca entre estabelecimentos comerciais e seus clientes. Diante dos achados foi possível confirmar a segunda hipótese (H2) investigada neste estudo. Ou seja, quanto maior a frequência de uso dos serviços disponibilizados pelo centro de beleza, o cliente passa a perceber mais fortemente a força do relacionamento desenvolvida junto ao prestador de serviço.

A associação entre características socioeconômicas e demográficas e a variável dependente antes citada foi parcialmente confirmada (H3). Em suma, os achados da presente pesquisa não possibilitam afirmar que a renda do cliente (H3a), 
a idade (H3b), bem como a distância entre a residência deste e o centro de beleza que frequenta, afetam a força do relacionamento desenvolvida entre consumidores e centros de beleza.

Foi possível perceber, entretanto, que existe uma associação linear significativa entre sexo do respondente e a força da relação empresa-cliente. Diante disso, infere-se que a maior parte das mulheres inquiridas percebe que se relacionam fortemente com seus centros de beleza. Já a maior parte dos respondentes do sexo masculino confessa que não possui qualquer tipo de relacionamento com os salientados prestadores. Diante desse achado foi possível confirmar a H3c deste estudo.

Com base em Souza Neto e Mello (2009), com relação à natureza das relações empresa-cliente, é possível que as mulheres inquiridas tendam a interagir com seu centro de beleza de forma mais frequente, por um longo prazo e de difícil rompimento quando comparadas aos respondentes do sexo masculino.

Por fim, ainda foi possível averiguar que os Aspectos de Competências Individuais (Empatia, Reciprocidade e Confiabilidade) e, em especial, os Profissionais (Competência para reconhecer a importância do cliente, Capacidade de ser atencioso, Compromisso com as atividades desenvolvidas e Respeito aos valores dos clientes), impactam na promoção da força da relação empresacliente. Ante o fato citado, é possível deduzir que, para a amostra em questão, quanto maior a percepção do cliente acerca da importância dos atributos de relacionamento desenvolvidos com seu fornecedor, maior será a força da relação desenvolvida entre eles. Tal achado confirmou a quarta hipótese (H4) proposta por este estudo, ou seja, a percepção do cliente acerca da importância dos atributos de relacionamento desenvolvidos pelo seu fornecedor influenciará na força da relação desenvolvida entre eles.

\section{Referências}

ADJEI, M. T.; CLARK, M. N. Relationship marketing in A B2C Context: The moderating role of personality traits. Journal of Retailing and Consumer Services, 2010.

ALVES, M. T. G.; SOARES, J. F. Medidas de nível socioeconômico em pesquisas sociais: uma aplicação aos dados de uma pesquisa educacional.Opin.

Publica,Campinas, v. 15,n. 1,June 2009.

BARNES, J. Closeness, Strenght and Satisfaction: Examining the Nature of Relationships between Providers of Financial Services and their Retail Customers, Psychology and Marketing, v. 14, n. 8, p. 765-790, 1997.

KUMAR, V; BOHLING, T. R.; LADDA, R. N. (2003). Antecedents and consequences of relationship intention: Implications for transaction and relationship marketing. Industrial Marketing Management,v. 32, n. 8, p. 667-676, 2003.

\section{BARNES, J. G. Segredos da gestão pelo} relacionamento com os clientes: é tudo uma questão de como você faz com que eles se sintam. Rio de Janeiro: Qualitymark, 2002.

BARNES, J.G. Exploring the importance of closeness in customer relationships. American Marketing

Association, v. 6, p. 227-40, 1997.

BARRY, J. M.; DION, P.; JOHNSON, W. A crosscultural examination of relationship strength in $\mathrm{B} 2 \mathrm{~B}$ services, Journal of Services Marketing, v. 22, n. 2, p.114-135, 2008.

BASÍLIO, J. C. Hair Brasil 2014 registrou momento histórico para o segmento: lançamento da primeira norma técnica do País para os serviços prestados pelos salões de beleza. Disponível em: http:/ /www. abihpec.org.br/. Acessado em: 03 jun. 2016.

BESSEGATO, A. M.; NEUHAUS; M. C. O marketing de relacionamento na Cerealista Giruá Ltda. In: VIII SEMEAD, 2005, São Paulo. Anais... São Paulo: SEMEAD, 2005.

CERQUEIRA, C. A. ; GIVISIEZ, G. H. N. . Conceitos básicos em demografia e dinâmica demográfica brasileira. In: Eduardo Luiz G. Rios-Neto; Juliana de Lucena RuasRiani. (Org.). Introdução à demografia da educação. 1 ed. Campinas: Associação Brasileira de Estudos Populacionais - ABEP, 2004, p. 13-44. 
COOIL, B., KEININGHAM, T. L; AKSOY, L.; Hsu, M. A Longitudinal Analysis of Customer Satisfaction and Share of Wallet: Investigating the Moderating Effect of Customer Characteristics, Journal of Marketing, v. 71 (January), p. 67-83, 2007.

CRESWELL, J. W. Projeto de Pesquisa: métodos qualitativo, quantitativo e misto. Tradução Magda Lopes; consultoria, supervisão e revisão técnica desta 3a edição Dirceu da Silva. Porto Alegre: Artmed, 2010, 296 p.

DAGGER, T; DANAHER, P. J.; GIBBS, B. How Often versus How Long: The Interplay of Contact Frequency and Relationship Duration in Customer Perceptions of Service Relationship Strength, Journal of Service Research, v. 11, n. 4, p. 371-388, 2009.

D’ANGELO, A. C.; SCHNEIDER, H.; LARAN, J. A. Marketing de Relacionamento junto a consumidores finais: um estudo exploratório com grandes empresas brasileiras. Revista de Administração Contemporânea, v. 10, n. 1, p. 73-93, 2006.

DEMO, G. Políticas de gestão de pessoas nas organizações: o papel dos valores pessoais e da justiça organizacional. 3. ed. São Paulo: Atlas, 2010.

DEMO, G.; PONTE, V. Marketing de Relacionamento (CRM): estado da arte e estudo de casos. São Paulo: Atlas, 2008.

Demo, G. B2C market: development of a CRM scale. In A. Ghorbani (Ed.). Marketing in the

Cyber Era (pp. 85-95). Hershey, PA: IGI Global, 2014.

FORD, D. L. E.; GADDE, H. H; SNEHOTA, I. Managing Business Relationships. Chichester: John Wiley \& Sons, 2003.

FROEMMING, L. Encontros de Serviços em uma Instituição de Ensino Superior. 284 f ., Tese Doutorado em Administração de Empresas, Universidade Federal do Rio Grande do Sul, Porto Alegre, 2001.

Gadde, L. E.; Huemer, L.; Hakansson, H. Strategizing in industrial networks Industrial Marketing Management, v. 32, n. 5, p. 357-64, 2003.

GARBACCIO, J. L.; OLIVEIRA, A. C. de. O risco oculto no segmento de estética e beleza: uma avaliação do conhecimento dos profissionais e das práticas de biossegurança nos salões de beleza. Texto Contexto Enferm, v. 22, n. 4, p. 989-98, 2013.
GRAZIANO, G. O.; OSWALDO, Y. C. ; PIZZINATTO, A. K. ; SPERS, V. R. E. Programas de Endomarketing na Indústria de Produtos Orgânicos: Estudo do Marketing de Relacionamento. REMark. Revista Brasileira de Marketing, v. 15, n. 5, p. 626-641, 2016.

GRONROOS, C. The relationship marketing process: Communication, interaction, dialogue, value. Journal of Business and Industrial Marketing, v. 19, n. 2, p. 99113, 2004.

GWINNER, K., GREMLER, D.; BITNER, M. J. Relational benefits in services industries: the customer's perspective. Journal of the Academy of Marketing Science, v. 26, n. 2, p. 101-14, 1998.

HAUSMAN, A. Variations in relationship strength and its impact on performance and satisfaction in business relationships. Journal of Business \& Industrial Marketing, v. 16, n. 7, p.600-616, 2001.

HOLMLUND, M. Perceived Quality in Business Relationships. Helsink: Swedish School of Economics and Business Administration Library, 2000.

KUMAR. V.; JONES, E.; VENKATESAN, R.; LEONE, R. P. Is market orientation a source of sustainable competitive advantage or simply the cost of competing? Journal of Marketing, v. 75, n.1, p. 16-30, 2011.

LADHARI, R. A review of twenty years of SERVQUAL research. International Journal of Quality and Service Sciences, v. 1 n. 2, p. 172-198, 2009.

LARENTIS, F.; ANTONELLO, C. S. ; MILAN, G. S. ; DE TONI, D. Aprendizagem Organizacional e Relacionamentos Interorganizacionais: um estudo de casos múltiplos. Revista Base , v. 11, p. 347-366, 2014.

Lovelock, C.; Patterson, P.; Walker, R. Services

Marketing: An Asia-Pacific and Australian Perspective. French Forest, New South Wales: Prentice Hall, 2004.

MIYAMOTO, T.; REXHA, N. Determinants of three facets of customer trust: a marketing model of Japanese buyer-supplier relationship, Journal of Business

Research, v. 57, n. 3, p. 312-9, 2004.

MORGAN, R. M.; HUNT, S. D. The CommitmentTrust Theory of Relationship Marketing Journal of Marketing, p. 20-38, 1994.

MOTA, M. de O.; FREITAS, A. A. F. Análise dos Benefícios Relacionais Observados por Usuários de Serviços. RAM. Revista de Administração Mackenzie, v. 9, set/out 2008, 126-147. 
NG, S.; DAVID M.E; DAGGER, T.S. Antecedents and consequences of customer - service provider relationship strength. International Journal of Business

Environment, v. 5, n. 3, p. 232-251, 2013.

NICHOLSON, C. Y., COMPEAU, L. D.; SETHI, R. The Role of Interpersonal Liking in Building Trust in LongTerm Channel Relationships, Journal of the Academy of Marketing Science, Vol. 29, n.1, p. 3-13, 2001.

NICKELS, W. G.; WOOD, M. B. Marketing: relacionamento, qualidade, valor. Rio de Janeiro: LTC, 1999.

O'MALLEY, L.; TYNAN, C. (2000). Relationship Marketing in Consumer Markets: Rhetoric or Reality?, European Journal of Marketing, v. 34, n. 7, p. 797-815, 2000.

PALMATIER, R. W.; DANT, R. P.; GREWAL, D.; Evans, K. R. Factors Influencing the Effectiveness of Relationship Marketing: A Meta- Analysis, Journal of Marketing, v. 70 (October), p. 136-153, 2006.

PARVATIYAR, A.; SHETH, J. N. The domain and conceptual foundations of relationship marketing. Handbook of relationship marketing. Sage

Publications. Thousands OAKS, 2000.

PAYNE, A. Handbook of CRM: achieving excellence in customer relationship management. Oxford: Elsevier, 2006.

REINARTZ, W. J.; KUMAR, V. (2000). On the profitability of long-life customers in a non-contractual setting: An empirical investigate ion and implications for marketing. Journal of Marketing, v. 64, n.4, p. 17-35, 2000.

REIS, A. C. B. C. ; IACOVELO, M. T.; ALMEIDA, L. B. B. ; COSTA FILHO, B. A. . Marketing de relacionamento: agregando valor ao negócio com big data. REMark.

Revista Brasileira de Marketing, v. 15,n. 4, p. 512-523, 2016.

SCHEER, L. K., MIAO, C. F., \& PALMATIER, R. $\mathrm{W}$. Dependence and interdependence in marketing relationships: Meta-analytic insights. Journal of the Academy of Marketing Science, v. 43, n. 6, P. 694-712, 2015.

SHETH, J. N.; PARVATIYAR, A. E. The evolution of relationship marketing. In J. N. Sheth \& A. E. Parvatiyar (Eds.), Handbook of Relationship Marketing.

London: Sage Publications, 2000.
SHETH, J. N; PARVATIYAR, A. Evolving relationship marketing into a discipline. Journal of Relationship Marketing, vol. 1 n. 1, p. 3-16, 2002.

SOUZA NETO, A. F. de; MELLO, S. C. B. de. Características dos relacionamentos estabelecidos em diferentes contextos de serviços sob a ótica do consumidor. Revista de Administração de Empresas., São Paulo, v. 49, n. 3, set. 2009.

TRACEY, S.; DAGGER, M. E.; DAVID, S. N. Do relationship benefits and maintenance drive commitment and loyalty? Journal of Services Marketing, v. 25, n. 4, p. $273-281,2011$.

WARD, T.; DAGGER, T. S. The complexity of relationship marketing for service customers. Journal of Services Marketing, v. 21, n. 4, p. 281-290, 2007.

ZEITHAML, V. e BITNER, M. Marketing de Serviços: A Empresa com Foco no Cliente. Bookman, Porto Alegre, 2003. 